\section{Abszedierende Pneumonie durch Pseudomonas aeruginosa als Berufskrankheit bei einem Bohrwerksdreher}

L. Zell'1, U. Mack², A. Sommerfeld ${ }^{1}$, A. Buchter ${ }^{1}$, G. W. Sybrecht ${ }^{2}$

${ }^{1}$ Institut und Poliklinik für Arbeitsmedizin der Universität des Saarlandes, Homburg

(Leiter: Prof. Dr. med. A. Buchter)

2 Medizinische Universitätsklinik, Innere Medizin V (Pneumologie), Homburg

(Direktor: Prof. Dr. med. G. W. Sybrecht)
Zusammenfassung: Es wird ein 29jähriger immunkompetenter Patient mit einer ambulant erworbenen abszedierenden Pneumonie beschrieben, wobei als Erreger Pseudomonas aeruginosa identifiziert werden konnte. Infektionsquelle war das mit einem wasserlöslichen Kühlschmierstoff versetzte Bohrwasser, welches mit Pseudomonas aeruginosa kontaminiert war. Eine nachgewiesene Sensibilisierung im Sinne einer Allergiereaktion vom Typ III auf Pseudomonas aeruginosa fand sich als Hinweis auf eine längerfristige inhalative Exposition gegenüber dem kontaminierten Bohrwasseraerosol. Es erfolgte eine Anzeige der Pneumonie des Patienten als Berufskrankheit an den Unfallversicherungsträger mit Diskussion der Zusammenhangsfrage und der Empfehlung expositionsmindernder Präventivmaßnahmen.

Metalworking fluid-associated community-acquired pneumonia with lung abscess caused by Pseudomonas aeruginosa as an occupational disease: We report the case of a 29year-old man without immunodeficiency who acquired Pseudomonas aeruginosa pneumonia complicated by pulmonary abscess. The source of infection could be identified as aerosolized metalworking fluid at his workplace contaminated with Pseudomonas aeruginosa. A high titer of specific IgG antibodies (type-III-sensitization, Gell \& Coombs) against Pseudomonas aeruginosa has been identified in the patients serum as an indicator for longstanding occupational airborne exposure to contaminated metalworking fluid. This community-acquired pneumonia has been reported to the industrial injuries insurance as an occupational disease for discussion of legal consequences and development of effective measures of prevention.

\section{Einleitung}

Eine ambulant erworbene Pneumonie beim immunkompetenten jungen Erwachsenen ohne wesentliche Vorerkrankungen durch den Erreger Pseudomonas aeruginosa stellt eine Seltenheit dar [3] und muß zu Nachforschungen über mögliche Ursachen und Infektionswege Anlaß geben. Im folgenden wird ein Patient beschrieben, der aufgrund seiner beruflichen Tätigkeit als Bohrwerksdreher eine langfristige inhalative Exposition gegenüber einem mit Pseudomonas aeruginosa kontaminierten Bohrwasseraerosol hatte und mit dem Krank-

Pneumologie 53 (1999) 620-625

(c) Georg Thieme Verlag Stuttgart · New York heitsbild einer abszedierenden Pneumonie stationär behandelt wurde. Die unfallversicherungsrechtliche Fragestellung nach der Bewertung als Berufskrankheit wird diskutiert.

\section{Falldarstellung}

\section{Allgemeine Anamnese}

29jähriger Patient mit seit über zwei Jahren gehäuften, teilweise febrilen Infekten (Sinusitiden, Bronchitiden), mehrfache antibiotische und auch alternativmedizinische Therapien. Gewichtsabnahme von $12 \mathrm{~kg}$ in den letzten Monaten (aktuell $63 \mathrm{~kg}$, Größe $180 \mathrm{~cm}$ ). Akute stationäre Aufnahme bei ausgeprägter Infektsymptomatik mit Husten, gelblichem Auswurf, Fieber $\left(39,5^{\circ} \mathrm{C}\right)$, linksthorakalen Schmerzen und Kollapszustand. Unauffällige Vorgeschichte bis auf Appendektomie und Tonsillektomie. Zigarettenkonsum etwa zehn packyears, zuletzt fünf Zigaretten/die.

\section{Berufsanamnese}

Nach Schulabschluß Lehre als Bohrwerksdreher in einer Maschinenfabrik. Anschließend Anstellung im erlernten Beruf in einer großen Stahl- und Apparatebaufirma über neun Jahre bis zum aktuellen Krankheitsgeschehen. Dort ist der Patient zuletzt seit vier Jahren kontinuierlich an einem CNC-Bohrwerk ohne Kapselung und ohne Absaugung eingesetzt (Abb.1). Das mit Kühlschmierstoffen versetzte Bohr- bzw.

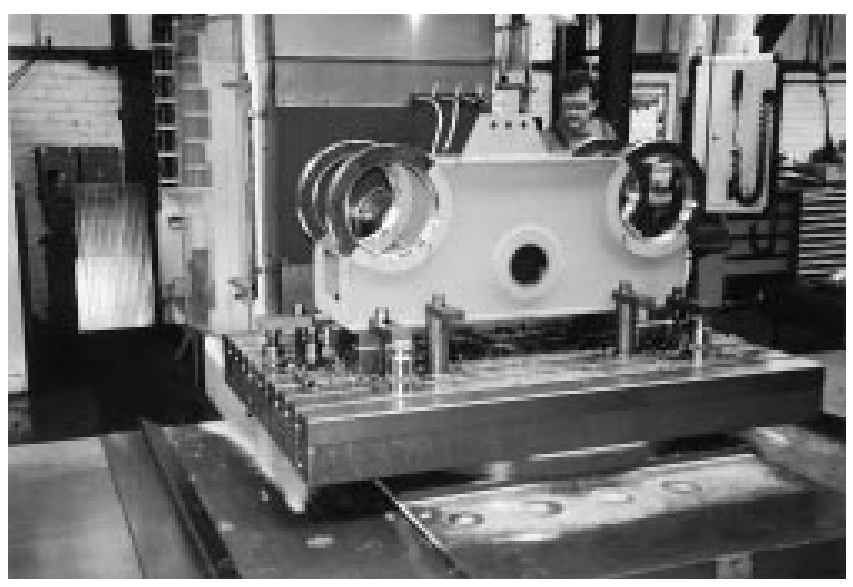

Abb. 1 Arbeitsplatz des Patienten (Bohrwerk) ohne technische oder persönliche Schutzmaßnahmen zur Verminderung der Aerosolexposition. 


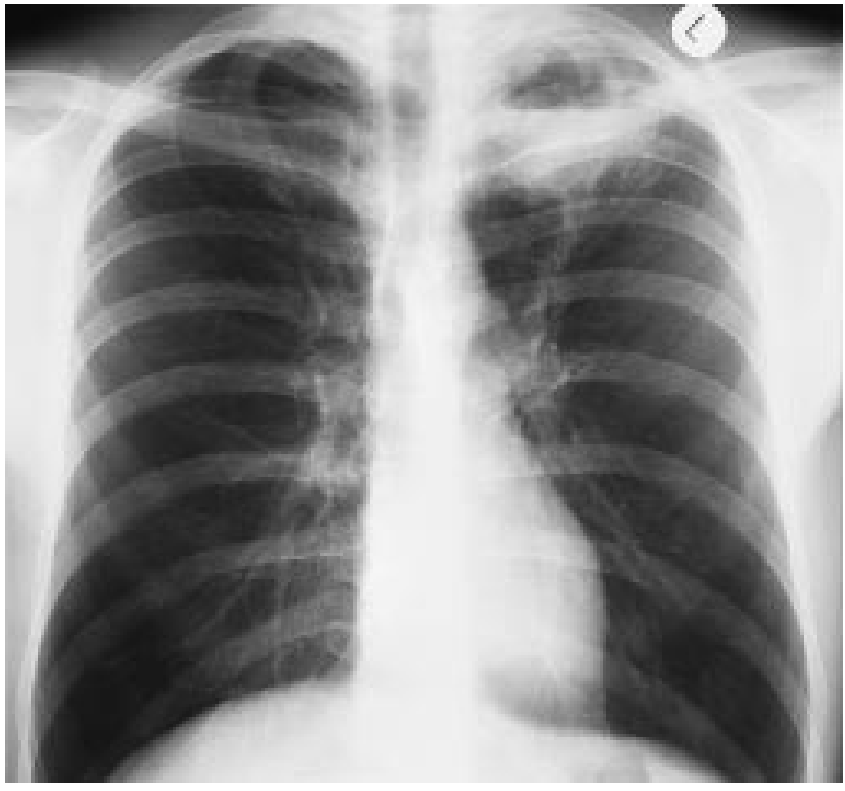

Abb. 2 Röntgen-Thorax zu Beginn der antibiotischen Therapie.

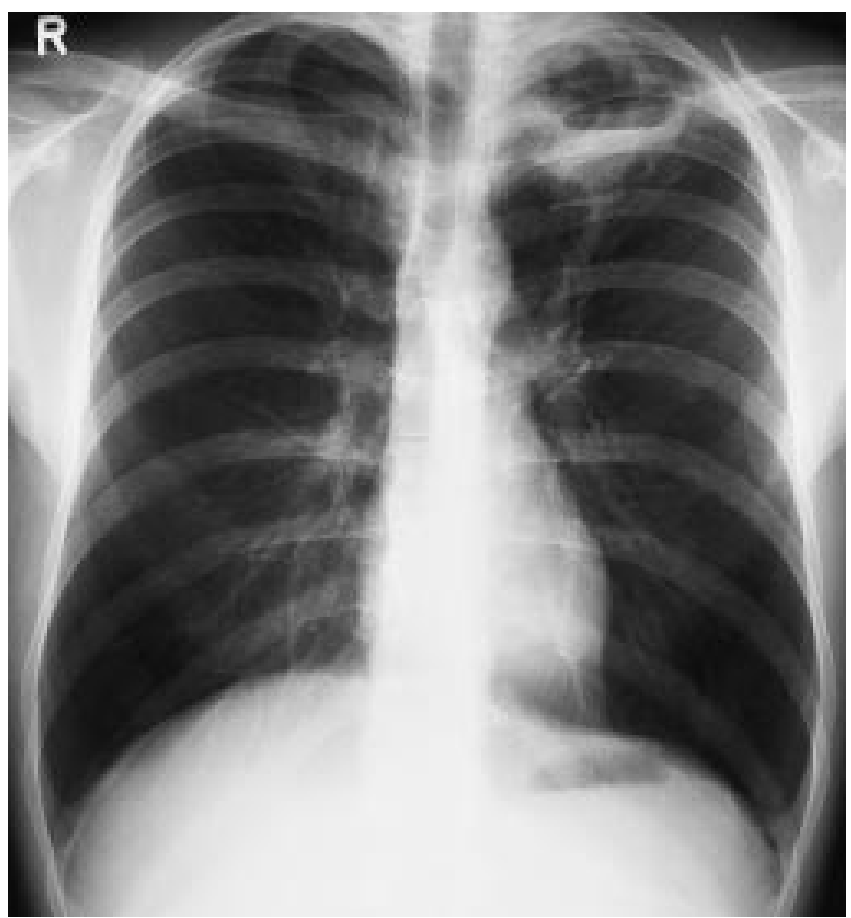

Abb. 3 Röntgen-Thorax nach zweiwöchiger antibiotischer Therapie.

Schleifwasser zeige nach anamnestischen Angaben in den Ablaufrinnen und Wannen gelegentlich eine Schimmelpilzbesiedlung. Durch die Dreh- bzw. Bohr- und Schleifvorgänge komme es, verstärkt durch Nachbarmaschinen, ständig zu deutlicher "Nebelbildung“ in der Arbeitshalle. Es bestehe eine erhebliche Bohrwasseraerosolexposition und -inhalation.

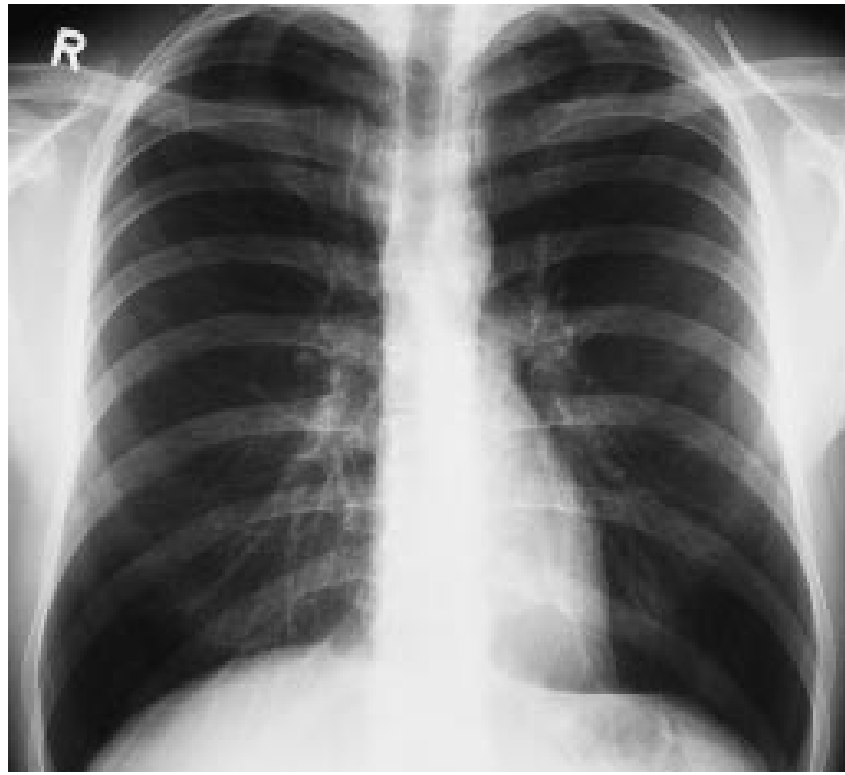

Abb. 4 Röntgen-Thorax sieben Wochen nach Therapiebeginn.

\section{Klinischer Befund}

Reduzierter Allgemeinzustand, blasse Haut und Schleimhäute. Kardiopulmonal keine auskultatorischen Auffälligkeiten.

\section{Laboruntersuchungen}

An Entzündungszeichen initial BSG 96/104 mm n.W., Leuko-

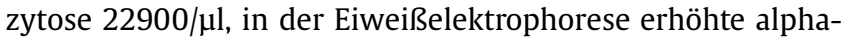
2-Globulinfraktion. Hämoglobin $11,3 \mathrm{~g} / \mathrm{dl}$. In der infektionsserologischen Diagnostik HIV-Serologie negativ, CMV-Diagnostik negativ, Legionellen-, Chlamydien- und Mycoplasmenserologie negativ. Kein pathologischer Nachweis von Autoantikörpern.

\section{Bildgebende Verfahren}

In der Röntgenübersichtsaufnahme des Thorax zunächst milchig trübes Infiltrat im linken Oberfeld mit Luft-Flüssigkeits-Spiegel (Abb.2). Unter antibiotischer Therapie Regredienz der Verschattung (Abb. 3) und zuletzt vollständige Auflösung (Abb. 4). In der thorakalen Computertomographie Darstellung einer etwa $3 \times 2,5 \times 2,5 \mathrm{~cm}$ großen zentral einschmelzenden unregelmäßig begrenzten Struktur im apikalen Oberlappensegment links mit Anschluß an das Bronchialsystem (Abb. 5-8).

\section{Flexible Bronchoskopie}

Gerötete und ödematöse Schleimhaut LB 1 und 2, wenig weiß-gelbliches Sekret LB 2.

\section{Mikrobiologische Untersuchungen}

In Blutkulturen, im Bronchialaspirat und im Sputum mehrfacher Nachweis von Pseudomonas aeruginosa. 

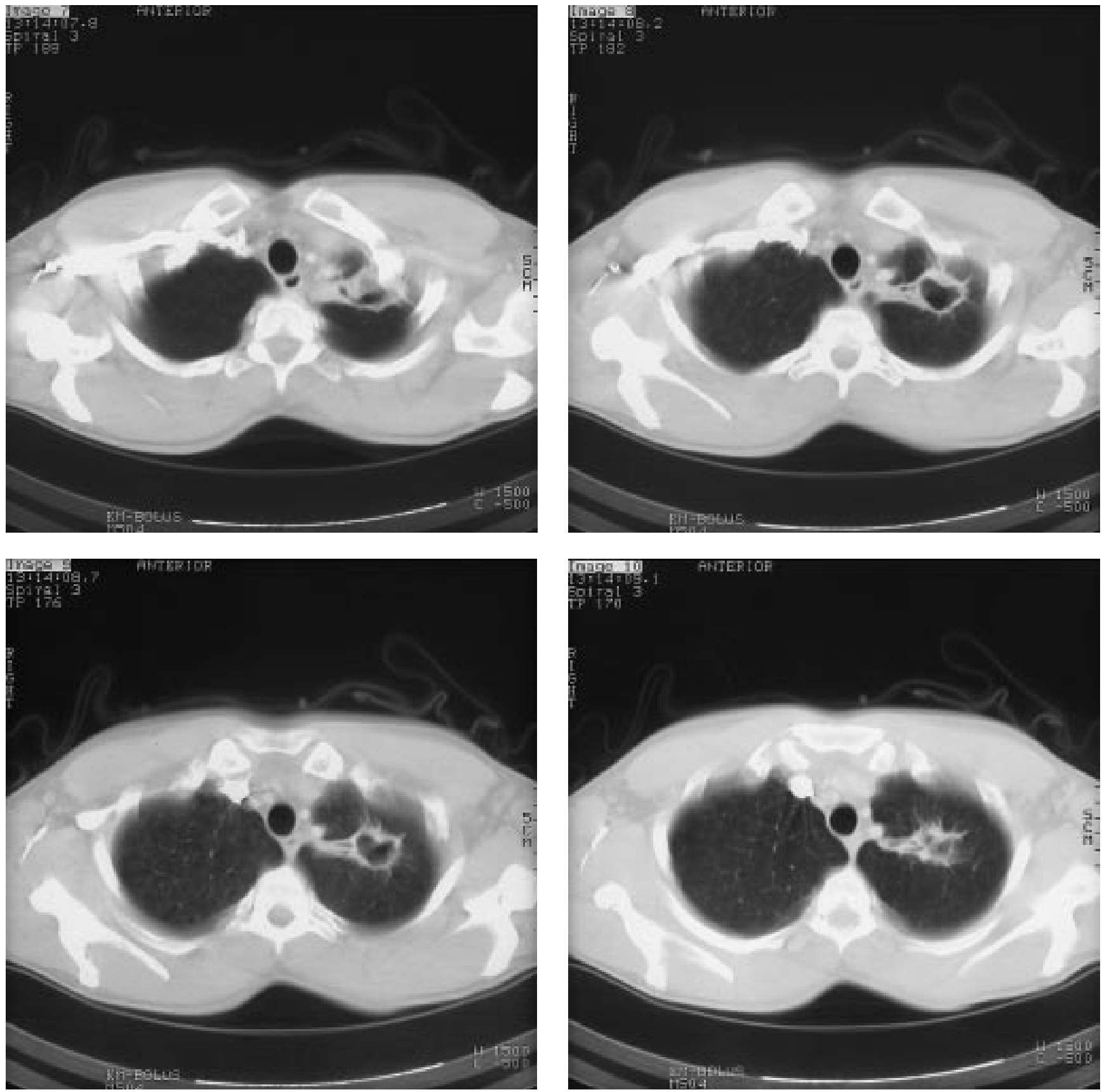

Abb. 5-8 Thorax-Computertomographie zwei Wochen nach Beginn der antibiotischen Therapie mit gut erkennbarer Abszeßhöhle im linken Oberlappen.

\section{Weitere Untersuchungen}

Bei Aufnahme blutgasanalytisch Hyperventilation. In der Bodyplethysmographie erhöhtes Residualvolumen $(130 \%$ des Solls) und diskrete Obstruktionszeichen in der Fluß-VolumenKurve, keine restriktive Ventilationseinschränkung. Unauffälliges EKG. Echokardiographie ohne pathologischen Befund.

\section{Verlauf}

Durchführung einer Antibiose nach Antibiogramm mit Ceftazidim und Tobramycin, dabei Regredienz des Infiltrates und der Abszeßbildung im linken Oberlappen.

\section{Arbeitsmedizinisches Procedere}

Besichtigung des Arbeitsplatzes und der CNC-Bohrwerkmaschine des Patienten einschließlich Entnahme von Bohrwasserproben und anschließende mikrobiologische Aufarbeitung dieser Proben mit Nachweis verschiedener Keime einschließ- 
lich Pseudomonas aeruginosa (Tab.1). Anschließend Untersuchung von Patientenserum auf das Vorliegen spezifischer IgG-Antikörper gegenüber den Keimanzüchtungen aus den Bohrwasserproben und Nachweis einer eindeutigen Sensibilisierung des Patienten auf Pseudomonas aeruginosa im Sinne einer Allergiereaktion vom Typ III (Tab.1) als Zeichen einer längerfristigen Exposition gegenüber Pseudomonas aeruginosa über die Inhalation des Bohrwasseraerosols.

Tab. 1 Keimnachweis aus Bohrwasserproben und IgG-Werte des Patienten in bezug auf die isolierten Keime

\begin{tabular}{ll}
\hline Isolierter Keim & IgG-RAST-Antikörperbestimmung \\
\hline Pseudomonas aeruginosa & $148,0 \%(!)$ \\
Moraxella lacunata & $94,5 \%$ \\
Pseudomonas alcaligenes & $6,6 \%$
\end{tabular}

(IgG-Werte: < 40\% Exposition nicht erkennbar, 40-100\% Exposition möglich, $>100 \%$ positiver Expositionshinweis)

Aufgrund des direkten Nachweises von Pseudomonas aeruginosa im Bohrwasser, angesichts der Sensibilisierung des Patienten als Hinweis für eine längerfristige Exposition gegenüber einem mit Pseudomonas aeruginosa kontaminierten Bohrwasseraerosol, wie auch aufgrund des Nachweises von Pseudomonas aeruginosa in den Blutkulturen, dem Sputum und dem Bronchialaspirat des Patienten Erstellung einer Berufskrankheitenanzeige an den zuständigen Unfallversicherungsträger (gemäß Berufskrankheitenziffer 3101: „Infektionskrankheiten, wenn der Versicherte im Gesundheitsdienst, in der Wohlfahrtspflege oder in einem Laboratorium tätig oder durch eine andere Tätigkeit der Infektionsgefahr in ähnlichem Maße ausgesetzt war“).

\section{Diskussion}

In der Erregerdiagnostik bei ambulant erworbener Pneumonie sind neben Streptococcus pneumoniae insbesondere Haemophilus influenzae und Viren sowie die Gruppe der atypischen Erreger zu finden [3]. In der hier beschriebenen Kasusitik eines jungen immunkompetenten Mannes ohne bedeutende pulmonale oder sonstige Vorerkrankungen konnte bei dem Krankheitsbild einer abszedierenden Pneumonie in mehreren mikrobiologischen Untersuchungen (Blutkulturen, Sputum, Bronchialaspirat) Pseudomonas aeruginosa als ungewöhnliche Ursache nachgewiesen werden. Bei der Suche nach möglichen Infektionsquellen ließen sich mehrere Keime einschließlich Pseudomonas aeruginosa im Bohrwasser am Arbeitsplatz des Patienten, welcher seit mehreren Jahren ohne Wechsel an einem Bohrwerk in einer Stahl- und Apparatebaufirma tätig ist, isolieren. Nun stellte sich die auch zum Schließen einer Beweiskette unfallversicherungsrechtlich bedeutsame Frage, ob lediglich zum Zeitpunkt der Probenahme des Bohrwassers (mehrere Wochen nach stationärer Aufnahme des Patienten) eine zufällige Kontamination mit Pseudomonas aeruginosa bestand, oder ob auch parallel zum Beginn der Erkrankung oder vorher eine solche mikrobielle Verunreinigung vorlag. Da entsprechende regelmäßige mikrobiologische Untersuchungen über die Bohrwasserqualität und Keimbesiedlungen in der Vergangenheit nicht durchgeführt worden waren, untersuchten wir Serum des Patienten hinsichtlich einer Sensibilisierung im Sinne einer Allergiereaktion vom Typ III auf das Vorliegen spezifischer Antikörper vom Typ IgG gegenüber Pseudomonas aeruginosa und auch den weiteren im Bohrwasser nachgewiesenen Keimen. Hierbei fand sich eine deutliche IgG-Erhöhung gegenüber Pseudomonas aeruginosa als Hinweis für eine Exposition, welche damit längerfristig vorbestanden haben mußte. Damit konnte eine berufskrankheitenrechtlich hinreichende Beweiskette im Sinne des Wahrscheinlichkeitsbeweises (Nachweis von Pseudomonas aeruginosa in Blutkulturen, Bronchialaspirat und Sputum - direkter Nachweis von Pseudomonas aeruginosa im Bohrwasser - Sensibilisierung des Patienten im Sinne einer Allergiereaktion Typ III gegenüber Pseudomonas aeruginosa durch längerfristige inhalative Bohrwasseraerosolexposition) geschlossen werden (Abb.9). Im vorliegenden Fall stellt sich retrospektiv außerdem die Frage, ob die vom Patienten anamnestisch geschilderte rezidivierende und teilweise febrile Infektsymptomatik auch im Sinne von akuten Episoden einer exogen-allergischen Alveolitis zu interpretieren ist.

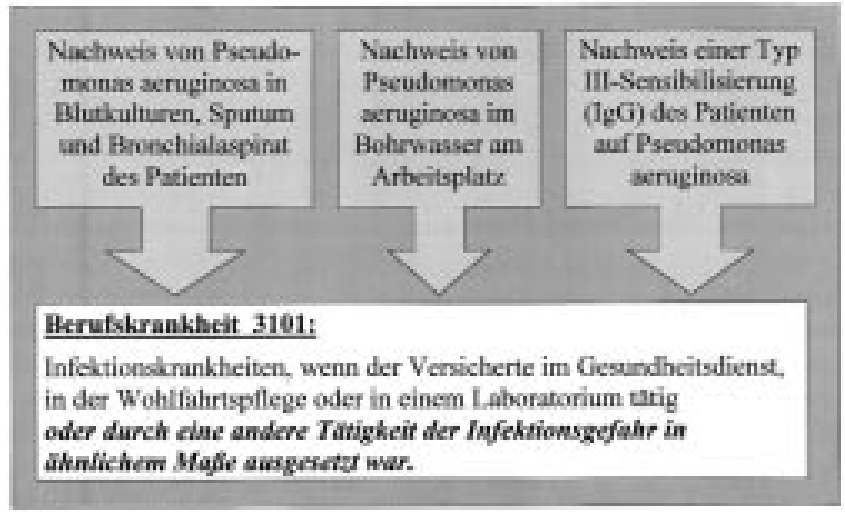

Abb. 9 Elemente der berufskrankheitenrechtlichen Beweisfrage zwischen Exposition und Krankheitsbild.

In der Literatur wurden bereits 1967 präzipitierende Antikörper gegenüber Pseudomonas fluorescens bei Arbeitern beschrieben, die Kontakt zu Kühlwasserrückständen bei Reinigungsarbeiten von Turbinenkesseln hatten, wo ebenfalls dieser Keim gefunden wurde [9]. Bei einer anderen Gruppe von sechs Arbeitern mit der Symptomatik einer exogenallergischen Alveolitis, welche alle eine Exposition gegenüber einem Aerosol eines wassermischbaren Kühlschmierstoffs hatten, wurden auch bei allen präzipitierende Antikörper ebenfalls gegen Pseudomonas fluorescens gefunden, dieser Keim war zusätzlich im Kühlschmierstoff nachweisbar [1,2]. Im Rahmen der Entstehung von Lungenfibrosen bei Hartmetallschleifern wurde der Nachweis präzipitierender Antikörper gegen verschiedene Pilze in einem Kollektiv gesunder Hartmetallschleifer als ätiologisch bedeutsam diskutiert [14]. In einem Fall wurde eine manifeste Lungenfibroseerkrankung infolge einer exogen-allergischen Alveolitis bei pilzkontaminiertem Schleifwasser dargestellt [16]. Bei Naßschleifern in der metallverarbeitenden Industrie konnte im Vergleich zu Trockenschleifern eine höhere IgG-vermittelte Sensibilisierung gegenüber verschiedenen Schimmelpilzen nachgewiesen werden, die Höhe der Sensibilisierung hing zusätzlich von der Beschäftigungsdauer ab [4]. Bei einer anderen Gruppe von 
Arbeitern, ebenfalls aus der metallverarbeitenden Industrie, die eine mindestens einjährige inhalative Exposition gegenüber einem Schleifwasseraerosol hatten, welches nachweislich in hoher Konzentration mit Pseudomonas pseudoalcaligenes kontaminiert war, fanden sich gegenüber dieser Pseudomonasspezies im Vergleich zu Kontrollgruppen (Blutspender, neu eingestellte Arbeitnehmer) signifikant erhöhte IgGSpiegel [7].

Pseudomonas aeruginosa ist als hygienisch relevante Keimverunreinigung in wassermischbaren Kühlschmierstoffen allgemein bekannt $[12,13,15]$. Neben den vielfältigen Möglichkeiten gesundheitlicher Beanspruchungen bei inhalativer Belastung durch Kühlschmierstoffaerosole [11] wurde bereits tierexperimentell beim Einsatz wassermischbarer Kühlschmierstoffe gezeigt, daß durch Inhalation eine Beeinträchtigung des Surfactant-Systems einschließlich einer Schädigung von Pneumozyten des Typs II stattfindet [10]. Es ist vorstellbar, daß eine solche Vorschädigung eine zusätzliche Prädisposition für die Entstehung einer Pneumonie bei der hier vorliegenden Aerosolexposition darstellen kann.

\section{Zur Frage der Berufskrankheit}

Hinsichtlich der Frage der Bewertung der Pneumonie des geschilderten Patienten als Berufskrankheit (Berufskrankheitenziffer 3101: „Infektionskrankheiten, wenn der Versicherte im Gesundheitsdienst, in der Wohlfahrtspflege oder in einem Laboratorium tätig oder durch eine andere Tätigkeit der Infektionsgefahr in ähnlichem Maße ausgesetzt war") müssen zunächst die Elemente der Definition der Berufskrankheit nach $\S 9$, Absatz 1, Sozialgesetzbuch (SGB) VII berücksichtigt werden: Berufskrankheiten sind Krankheiten, „die nach den Erkenntnissen der medizinischen Wissenschaft durch besondere Einwirkungen verursacht sind, denen bestimmte Personengruppen durch ihre versicherte Tätigkeit in erheblich höherem Grade als die übrige Bevölkerung ausgesetzt sind“ [5]. Die bereits genannte häufige Kontamination von Bohrund Schleifwässern mit Pseudomonaden sowie die auch im Vergleich zu Kontrollgruppen in der Literatur beschriebene höhere Sensibilisierung von exponierten Arbeitern entsprechen diesen Kriterien. Zusätzlich wird im Gesetzestext unter §9 Absatz 3 SGB VII weiter ausgeführt: „Erkranken Versicherte ... an einer in der Rechtsverordnung ... genannten Berufskrankheit ... und können Anhaltspunkte für eine Verursachung außerhalb der versicherten Tätigkeit nicht festgestellt werden, wird vermutet, daß diese infolge der versicherten Tätigkeit verursacht worden ist“ [5]. Bei im vorliegenden Patientenfall fehlenden außerberuflichen Infektionsquellen ergibt sich aus diesem Paragraphen somit eine weitere Bestätigung zur positiven Zusammenhangsbeurteilung. Im erläuternden Merkblatt zur Berufskrankheit 3101 wird außerdem ausgeführt, daß der Tatbestand der Berufskrankheit in Bereichen außerhalb des Gesundheitswesens, der Wohlfahrtspflege oder Laboratorien auch dann erfüllt sein kann, wenn der Versicherte „durch eine andere Tätigkeit der Infektionsgefahr in ähnlichem Maße besonders ausgesetzt war“. Dies besagt, daß „eine der versicherten Tätigkeit innewohnende besondere Infektionsgefährdung vorhanden sein muß“. Diese „erhöhte Ansteckungsgefahr kann auch in den ... hygienischen ... Verhältnissen des Tätigkeitsortes begründet sein, an dem der Versicherte arbeiten ... muß“ [8]. Da alle diese berufskrankheitenrechtlichen Forderungen im vorlie- genden Erkrankungsfall gegeben sind, ist eine Bewertung als Berufskrankheit vorzunehmen.

\section{Schlußfolgerungen}

Präventivmedizinisch ergibt sich für Arbeitsplätze mit Bohroder Schleifwasseraerosolexposition neben einer regelmäßigen Wasserhygiene (Wasserwechsel, Biozidanwendung, mikrobiologische Untersuchungen) die notwendige Überlegung, ob durch eine Kapselung entsprechender Maschinen die Wassernebelkonzentrationen $\mathrm{zu}$ vermindern sind [6]. Im konkreten Fall sind durch den Unfallversicherungträger solche Präventionsmaßnahmen nach $\S 3$ der Berufskrankheitenverordnung erforderlich, um der „Gefahr, daß eine Berufskrankheit entsteht, wiederauflebt oder sich verschlimmert“, entgegenzuwirken [8]. In vergleichbaren Erkrankungsfällen muß eine arbeitsmedizinische Genese differentialätiologische Berücksichtigung finden und vom behandelnden Arzt bei begründetem Verdacht auf das mögliche Vorliegen einer beruflich verursachten Infektion eine Anzeige an den zuständigen Unfallversicherungsträger oder den Gewerbearzt übermittelt werden.

\section{Literatur}

${ }^{1}$ Bernstein DI, Lummus ZL, Santilli G, Siskosky J, Bernstein IL. Machine operator's lung. A hypersensitivity pneumonitis disorder associated with exposure to metalworking fluid aerosols. Chest 1995; 108: $636-641$

2 Blanc P. Reflections of an armchair tribologist. The potential importance of "Machine operator's lung". Chest 1995; 108: $593-594$

${ }^{3}$ Brown PD, Lerner SA. Community-acquired pneumonia. Lancet 1998; 352: 1295 - 1302

${ }^{4}$ Galetke W, Borsch-Galetke E, Stalder K, Hering KG, Rabente T. Untersuchung von Schleifern zur Bedeutung von Allergien in Kühlschmierstoffen für die Entstehung von Lungenfibrosen. Verh Dtsch Ges Arbeitsmed 1994; 34: 163 - 169

${ }^{5}$ Gesetz zur Einordnung des Rechts der Unfallversicherung in das Sozialgesetzbuch (Unfallversicherungs-Einordnungsgesetz UVEG). Bundesarbeitsblatt Heft 10, 1996. 35

${ }^{6}$ Hands D, Sheehan MJ, Wong B, Lick HB. Comparison of Metalworking Fluid Mist Exposures from Machining with Different Levels of Machine Enclosure. Am Ind Hyg Assoc J 1996; 57: $1173-1178$

${ }^{7}$ Mattsby-Baltzer I, Edebo L, Järvholm B, Lavenius B. Serum antibodies to Pseudomonas pseudoalcaligenes in metal workers exposed to infected metal-working fluid. Int Arch Allergy Appl Immunol 1989; 88: $304-311$

${ }^{8}$ Mehrtens G, Perlebach E. Die Berufskrankheitenverordnung (BeKV). Handkommentar aus rechtlicher und medizinischer Sicht für Ärzte, Versicherungsträger und Sozialgerichte. Berlin: Erich Schmidt Verlag, 1999. 34. Lieferung

${ }^{9}$ Schümann-Vogt B, Bundschuh G. Antikörper gegen Pseudomonas fluorescens, eine Ursache des sogenannten „Kondensatorenfiebers“ bei Kesselreinigern. Dtsch Gesundheitsw 1967; 22: $133-136$

10 Stalder K, Ladany E, Kroll A, Pantke T. Tierexperimentelle Untersuchungen zur Gesundheitsbeeinträchtigung durch Ölnebel. Dortmund: Bundesanstalt für Arbeitsschutz, 1985

${ }^{11}$ Stork J, Heger M, Nöring R, Thamm H. Inhalative Belastung durch Kühlschmierstoffaerosole - arbeitsmedizinische Aspekte. Zbl Arbeitsmed 1995; 45: 228 - 236

12 Ström GB, Mälarstig B, Blomquist G. Purification of Water-Based Cutting Fluids. Methods Enzymol 1994; 228: 672 - 678 
${ }^{13}$ Warfolomeow I. Keimbelastung wassergemischter Kühlschmierstoffe. Die BG, 1998: 274 - 281

${ }^{14}$ Weber A, Hartung M. Eine Pilotstudie zum Vorkommen von präzipitierenden Antikörpern gegen Schimmelpilz- und Actinomyceten-Antigene in Serumproben von Hartmetallschleifern. Prax Klin Pneumol 1982; 36: 72 - 74

${ }^{15}$ Woskie SR, Virji MA, Kriebel D, Sama SR, Eberiel D, Milton DK, Hammond SK, Moure-Eraso R. Exposure Assessment for a Field Investigation of the Acute Respiratory Effects of Metalworking Fluids. I. Summary of Findings. Am Ind Hyg Assoc J 1996; 57: $1154-1162$

${ }^{16}$ Zell L, Sommerfeld A, Rentz K, Ukena D, Sybrecht GW, Buchter A. Lungenfibrose bei einem Hartmetallschleifer: EAA durch mikrobielle Schleifwasserkontamination. Pneumologie 1999; 53: S 73

\section{Dr. med. Lothar Zell}

Institut und Poliklinik für Arbeitsmedizin der Universität des Saarlandes

Am Forum 6

D-66424 Homburg

E-mail: amlzel@med-rz.uni-sb.de 